\title{
A Paget's disease Case of Breast without Underlying
}

\section{Carcinoma}

\author{
Hilal Ayvaz $\mathrm{H}^{1 *}$, Canpolat $\mathrm{F}^{2}$, Çevirgen Cemil $\mathrm{B}^{2}$, Gökçe $\mathrm{A}^{3}$ and \\ Gönül M² \\ ${ }^{1}$ Süleyman Demirel University Hospital, Dermatology Department, Turkey \\ ${ }^{2}$ Dışkapı Yıldırım Beyazıt Training and Research Hospital, Dermatology Clinic, Turkey \\ ${ }^{3}$ Dışkapı Yıldırım Beyazıt Training and Research Hospital, Pathology Clinic, Turkey
}

\section{Case Report \\ Volume 4 Issue 1}

Received Date: January 29, 2019

Published Date: March 25, 2019

DOI: $10.23880 /$ cdoaj-16000176

*Corresponding author: Havva Hilal Ayvaz, Süleyman Demirel University Hospital, Dermatology Department, Turkey, Tel: 5309505907; Email: drhhilalayvaz@gmail.com

\section{Abstract}

Paget's disease of the breast is a rare type of cancer of the nipple-areola complex which is characterized by eczematous lesions. It is often associated with an underlying in-situ or invasive carcinoma but it is not a must. Paget's disease might be misdiagnosed as nipple eczema and this may cause a delay in the diagnosis.

A 65-year old female patient admitted to our department with a 1-year history of erythema and swelling which started over the left nipple and spread to the areola. The patient used topical steroid treatment which was unsuccessful. In her medical history, there was total right mastectomy operation for diagnosed ductal carcinoma. The patient presented with no active palpable mass at left breast or lymph nodes on both sides at that moment. Dermatological examination revealed sharp bordered, erythematous, mildly squamated, $2 \times 3 \mathrm{~cm}$ in diameter, solitary plaque lesion located on left nipple. Radiological imaging showed no abnormalities. Histopathological examination and immunohistochemical staining were compatible with Paget's disease. Surgery was scheduled.

This case has been presented to lay emphasis on Paget's disease which may be misdiagnosed easily and to remind that it could be without concurrent underlying malignancy at all.

Keywords: Paget's disease; Mastectomy; Neoplasm

\section{Introduction}

Paget's disease of the breast is a rare disease that usually occurs in the form of an eczema-like lesion on the nipple, accounting for about $1-3 \%$ of all breast carcinomas [1]. It is characterized by the presence of large, pleomorphic cells with pale cytoplasm invading the nipple epidermis [2]. Although it often coexists with an underlying breast carcinoma, it rarely develops without an underlying malignancy. For this reason, it is not enough to look only at the radiological findings; this can lead to delay in the diagnosis [3]. Here we present a case of Paget's disease, which had previously undergone breast carcinoma but detected no underlying active malignancy. 


\section{Case Report}

A 65-year-old woman admitted to our outpatient clinic with complaints of redness and bulging that started from the left nipple for one year and spread to the surrounding area. She had previously received topical treatment with diagnosis of eczema and it did not benefit. In her medical history, she had right breast mastectomy 10 years ago due to ductal carcinoma. The patient stated that she had no rash on the breast at that time. Physical examination revealed no mass found in the left breast or active axillary lymphadenopathy in the patient with right mastectomy. In the examination a $2 \times 3 \mathrm{~cm}$ sharp limited, erythematous, slightly squamous solitary plaque on the left breast (Figures 1a-1b) was notted. No abnormal finding was detected in US, MRI and mammography of the left breast.
Whole-body bone scintigraphy was normal. Histopathological examination of the lesion showed solid nesting in the epidermis and atypical epithelial cells showing pagetoid spreading as individual cells (Figure 2). In immunohistochemical examination epithelial membrane antigen was (EMA) + , carcinoembryonic antigen was (CEA)+, Her-2 oncoprotein was (CerbB2)+, cytokeratin was (CK) 7+, S100 - (Figure 3). The patient was diagnosed with Paget's disease in the presence of clinical and histopathological findings. Nevertheless, considering the risk of recurrence, advanced modified radical mastectomy surgery was planned as a result of the joint decision-maker between the medical oncology and surgery departments.
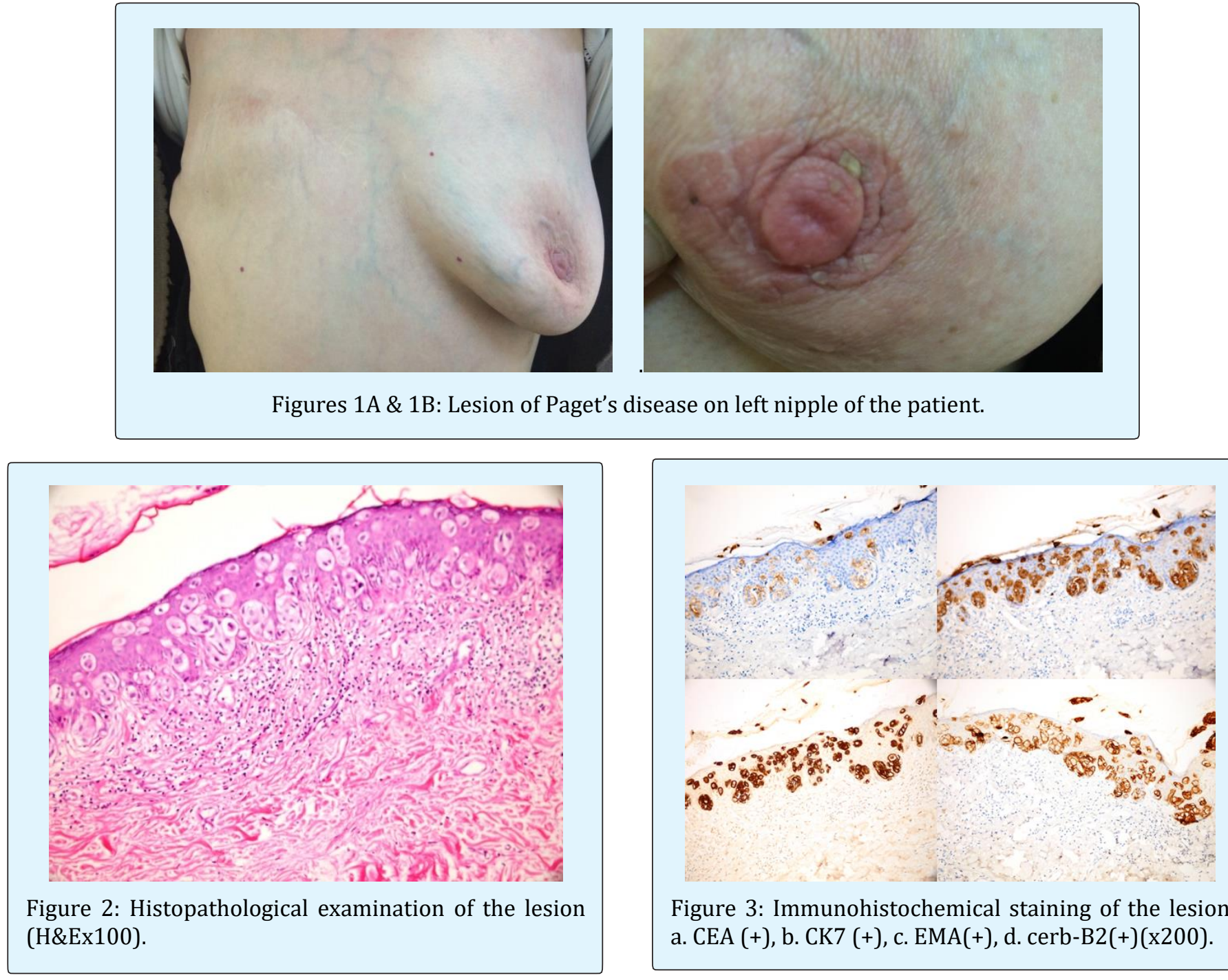

Figure 3: Immunohistochemical staining of the lesion a. CEA (+), b. CK7 (+), c. EMA(+), d. cerb-B2(+)(x200). 


\section{Clinical Dermatology Open Access Journal}

\section{Discussion}

Paget's disease is usually seen in the form of thickened, eczematoid, erythematous, and crustal lesions with irregular borders that spread from the breast to the areola and the surrounding area, mostly unilaterally $[4,5]$. Paget's disease lesions typically have a sharp discrimination with normal skin density, and the lesion has a slightly bulgy irregular appearance. Frequent complaints such as pain, burning, pruritus and sometimes bloody-pus discharge accompany. Nipple can be seen as retracted or deformed [6].

The incidence of Paget's disease among all breast cancer patients is about $0.5-5 \%$. It is usually seen postmenopausal and over 50 years of age in women, as in breast cancer [6,7]. Paget's disease is rarely seen in men as well. Although clinical and histopathological differences are not observed in male patients, Paget's disease may be worse in terms of progress [8].

$92-100 \%$ of the patients have underlying in-situ or invasive ductal carcinoma, approximately $50 \%$ of the patients have palpable mass. Rarely, this disease can develop without underlying malignancy [5]. Lymph node involvement and growth are also accompanied [6]. In our patient, there was no pathology, although the patient had a history of ductal carcinoma in the past.

There are two main theories in the pathogenesis of Paget's disease: The first epidermotropic theory claims that Paget cells originating from ductal cancer cells involve chemotaxis and invasion of the epidermis of the nipple. According to the second theory, which is more accepted, Paget cells are formed from in-situ malignant keratinocytes as a result of in-situ malign transformation or degeneration of the substantial cells [3].

Histopathological examination of the lesion reveals round, oval atypical Paget cells with a large, pleomorphic, hyperchromatic nucleus and a clear cytoplasm. These cells can be observed intraepidermally, individually or in a gravure pattern [5]. While almost all of the Paget cells are stained with CK7, they are not reactive with CK20. Paget cells are also stained with EMA, CEA, gross cystic disease liquid protein 15 (GCDFP-15), and some mucinous stains [9].S-100 and HMB45 are dyes used to distinguish Paget cells from in-situ melanomas. In Paget cases, which are mostly associated with underlying malignancy, they are positively stained with Cerb-B2. In our case there was: $\mathrm{EMA}+, \mathrm{CEA}+$, Cerb-B2 +, CK7 +, S100-. We think that the cause of Cerb-B2 staining being positive in our case may be related to ductal carcinoma that was previously present in other breast or undetected microscopic recurrence of carcinoma.

Topical steroids given for eczema diagnosis can cause some improvement in the inflammatory component of the disease and mask the underlying pathology for a while $[5,7]$. Other diseases that have differential diagnosis are benign intraductal papilloma, basal cell carcinoma, squamous cell carcinoma, superficial spreading malign melanoma, psoriasis, contact dermatitis, syphilis and Bowen's disease [5].

Although the diagnosis of the disease is made clinically, the radiographic examination should be performed to detect the underlying malignancy. Mammography may not always be helpful in the diagnosis of carcinoma insitus. In particular, it may be a more useful method in the presence of the mass $[6,9]$. Bilateral mammography is still an important method in the imaging of suspicious lesions and to investigate other breast. It should also be used for follow-up in the selected patients for conservative treatment [10]. US can be used for patients who are not observable with mammography but are suspicious. A more advanced and more sensitive method for patients with normal US and mammography is breast MRI [10]. Apart from these, 99mTc MIBI scintigraphy is another method that can be useful in showing intramedullary intraductal Paget's disease [3,5]. Moreover, patients with normal mammography or US findings, such as in our patients, may have delayed diagnosis only by looking at radiological findings. The detailed clinical examination is the most important step in the diagnosis of Paget's disease and definite diagnosis should be made both with radiological and histopathologic methods [5].

Mastectomy with or without lymph node is still considered standard therapy, but other methods such as breast-conserving surgery and local radiotherapy are alternatives [3].

In conclusion, the necessary and appropriate treatment for the patient should be selected after the clinical and radiological evaluations. The case was presented to emphasize that Paget's disease is an easily skipped diagnosis and, it may develop without the underlying malignancy.

Acknowledgment: There is no financial support at all. Authors are in agreement with the content of the manuscript and made equal contributions while the study was being prepared. 


\section{Clinical Dermatology Open Access Journal}

Conflict of Interest: There is no scientific or medical committee membership or consultancy, expertise, working status or shareholding in any firm related to this work, which might be a potential conflict of interest of authors and/or family members.

Consent Form: Permission from the patient was obtained to use the relevant information and photographs.

\section{References}

1. Kawase K, Dimaio DJ, Tucker SL, Buchholz TA, Ross MI, et al. (2005) Paget's disease of the breast: there is a role for breast-conserving therapy. Ann Surg Oncol 12(5): 391-397.

2. Fu W, Lobocki CA, Silberberg BK, Chelladurai M, Young SC (2001) Molecular markers in Paget disease of the breast. J Surg Oncol 77(3): 171-178.

3. Caliskan M, Gatti G, Sosnovskikh I, Rotmensz N, Botteri E, et al. (2008) Paget's disease of the breast: the experience of the European Institute of Oncology and review of the literature. Breast Cancer Res Treat 112(3): 513-521.
4. Rosen PP (2001) Paget's disease of the nipple. In: Rosen's breast pathology. $2^{\text {nd }}(E d n)$, Philadelphia: Lippincott-Raven, pp: 565-580.

5. Sakorafas GH, Blanchard K, Sarr MG, Farley DR (2001) Paget's disease of the breast. Cancer Treat Rev 27(1): 9-18.

6. Karakas C (2011) Paget's disease of the breast. Journal of Carcinogenesis 10: 31.

7. Kanitakis J (2007) Mammary and extramammary Paget's disease. J Eur Acad Dermatol Venereol 21: 581-590.

8. Akita M, Kusunoki N, Nakajima T, Takase S, Maekawa Y, et al. (2015) Paget's disease of the male breast: a case report. Surg Case Rep 1: 103.

9. Ikeda DM, Helvie MA, Frank TS, Chapel KL, Andersson IT (1993) Paget disease of the nipple: radiologicpathologic correlation. Radiology 189(1): 89-94.

10. Lim HS, Jeong SJ, Lee JS, Park MH, Kim JW, et al. (2011) Paget disease of the breast: mammographic, US, and MR imaging findings with pathologic correlation. Radiographics 31(7): 1973-1987. 